\title{
Traitement chélateur oral de la surcharge en fer
}

La surcharge en fer est une éventualité redoutable. Elle peut être aiguë, provoquée par une surdose accidentelle ou délibérée; plus fréquente est la surcharge chronique, qui revêt deux formes principales: l'hémochromatose congénitale, l'hémosidérose transfusionnelle. A long terme, les dépôts de fer saturent le système réticulo-endothélial, le fer se répand dans les parenchymes et le plasma sanguin; lorsque la transferrine, forme de transport du fer, est saturée puis débordée, le fer libre circule et exerce un pouvoir oxydant toxique. C'est dans la thalassémie majeure que les risques de surcharge sont les plus précoces, à la fois par absorption accrue et par apports transfusionnels. Ils aboutiraient à la mort dès l'adolescence, n'étaient deux traitements efficaces. Le premier, curatif, souvent définitivement, est la greffe de moelle osseuse ; mais son emploi est limité par des problèmes de compatibilité et de disponibilité. Le second, palliatif mais efficace, est l'injection d'un chélateur du fer, la desferrioxamine; elle présente deux avantages: la chélation, qui se fait molécule à molécule, est indépendante de la concentration en fer, et continue donc même si les réserves sont devenues plus basses; de plus, elle n'est pas toxique. En revanche, elle ne peut agir que par voie parentérale, obligeant donc à des injections quotidiennes pendant des années; coûteuse, elle est hors de portée de la plupart des habitants des pays pauvres. Son caractère contraignant fait que très souvent le traitement est mal suivi. On comprend que, depuis longtemps, on soit à la recherche d'un chélateur actif par voie orale. Les essais se sont concentrés sur un composé proposé en 1987 [1], appelé Ll puis défériprone. Chimiquement, il s'agit du 1,2-diméthyl-3-hydroxypyridine-4one. Ces essais sont toujours restés sans conclusion formelle, en raison d'effets toxiques sur la leucopoïèse (neutropénies et même cas d'agranulocytose qui se sont montrés réversibles). Toutefois, des auteurs originaires de pays en voie de développement ont continué à plaider en faveur du produit, arguant du fait qu'à son défaut la plupart des malades de ces pays n'étaient pas traités du tout $(\mathrm{m} / \mathrm{s}$ $n^{\circ} 5$ vol. 6, p. 491). Il était donc nécessaire de mettre en œuvre un essai correctement contrôlé. On ne connaît qu'imparfaitement le mode d'action de la défériprone: il faut trois molécules du produit pour lier une molécule de fer; de plus, l'affinité, contrairement à celle de la desferrioxamine, dépend de la concentration en fer. Il faut donc employer des doses élevées, proches des doses toxiques, et on ne peut espérer abaisser le taux des réserves au-dessous d'un certain seuil [2]. Une équipe de Toronto (Canada) et Cleveland $(\mathrm{OH}$, USA) [3] a entrepris un travail sur l'effet thérapeutique de la défériprone, chez des sujets thalassémiques ne prenant pas ou ne prenant plus de desferrioxamine, transf usés toutes les trois ou quatre semaines. Les auteurs ont considéré que la méthode habituelle d'évaluation des réserves en fer, le dosage de la ferritine sanguine, était trop indirecte et ne suffisait pas. Ils ont donc dosé directement le fer hépatique, soit par dosage chimique sur biopsie, soit par mesure magnétique in vivo, avec des résultats équivalents. Vingt et un malades ont été suivis pendant une moyenne de trois ans. La dose donnée était de $75 \mathrm{mg}$ par $\mathrm{kg}$, répartie chaque jour en trois prises. Le critère d'efficacité était l'évolution des réserves de fer du foie. Le niveau critique est considéré comme étant de $80 \mu \mathrm{mol} / \mathrm{g}$, audessous duquel on peut espérer l'absence de complications. On peut diviser les malades en deux groupes: dans l'un (dix sujets), le traitement antérieur par desferrioxamine avait été inefficace; chez eux, la concentration du fer est passée de $125 \pm 11$ à $60 \pm 10$ en moyenne. (hez les onze autres, l'abaissement obtenu antérieurement par le chélateur parentéral a été maintenu. Une baisse de la ferritinémie a été également constatée.

Les études de toxicité, dans ce groupe, n'ont montré aucune anomalie leucocytaire. L'analyse des fonctions immunologiques, entreprise en raison d'une atrophic thymique expérimentale chez des rongeurs, les a confirmées normales.

En conclusion, chez vingt et un malades, la défériprone a permis le maintien ou l'obtention d'un niveau des réserves hépatiques de fer compatible avec une survie prolongée dans des conditions satisfaisantes. Une anomalie apparente est que chez. environ la moitié des malades, un traitement antérieur par la desferrioxamine s'était montré inefficace; l'explication la plus probable est que ce traitement n'avait pas été correctement suivi, ce qui souligne à nouveau l'intérêt d'un traitement par voie orale, qui, lui, a semblé respecté dans presque tous les cas. Aucune complication grave n'a été notée ; mais l'échantillon de cette étude est trop petit ; l'existence de cas connus d'agranulocytose exige un essai plus étendu, multicentrique, en cours actuellement, comme préalable nécessaire à une autorisation de mise sur le marché. En attendant, les essais relatés autorisent beaucoup d'espoirs.

J.C.D.

1. Kontoghioghes (iJ, Aldouri MA, Sheppard I.N Hof fbrand AV. 1.2-dimethyl-3-hydroxypiridin-4 one: an orally active chelator for treatment of iron overload. loncel 1987 ; 1: 1294-5.

2. Nathan D(;. An orally active iron chelator. $N$ Engl J Med 1995; 3.32: 953-4.

3. ()livieri NF, Brittenham (BM, Matsui D), Berkovitch M, Blendis $1 . \mathrm{M}$, (ameron R(; Mcclelland RA, Liu PP, Templeton DM, Koren (;. Iron-chelation therapy with oral deferiprone in patients with thalassemia major. $N$ lingl / Med l995, 3.32: : 918-22. 